\title{
Cranberry Juice Ingestion and Clinical Drug-Drug Interaction Potentials; Review of Case Studies and Perspectives
}

Nuggehally R Srinivas

Suramus Biopharm, J.P. Nagar, I Phase, Bangalore 560078, India

Received, March 11, 2013; Accepted, June 17, 2013; Published, June 20, 2013.

\begin{abstract}
Cranberry juice is a popular beverage with many health benefits. It has anthocyanins to supplement dietary needs. Based on in vitro evidence cranberry juice is an inhibitor of CYP enzymes and at higher amounts as potent as ketoconazole (CYP3A) and fluconazole (CYP2C9). There is, however, a discrepancy between in vitro and in vivo observations with respect to a number of substrates (cyclosporine, warfarin, flurbiprofen, tizanidine, diclofenac, amoxicillin, ceflacor); with the exception of a single report on midazolam, where there was a moderate increase in the AUC of midazolam in subjects pre-treated with cranberry juice. However, another study questions the clinical relevancy of in vivo pharmacokinetic interaction between cranberry juice and midazolam. The controversy may be due to a) under in vitro conditions all anthocyanin principles may be available to have a concerted effort in CYP inhibition; however, limited anthocyanin principles may be bioavailable with varying low levels in the in vivo studies; b) a faster clearance of the active anthocyanin principles under in vivo conditions may occur, leading to low threshold levels for CYP inhibition; c) efficient protein binding and/or rapid tissue uptake of the substrate may have precluded the drug availability to the enzymes in the in vivo studies. With respect to pharmacodynamic aspects, while the debate continues on the issue of an interaction between warfarin and cranberry juice, the summation of the pharmacodynamics data obtained in patients and healthy subjects from different prospectively designed and controlled clinical trials does not provide overwhelming support for the existence of a pharmacodynamic drug interaction for normal cranberry juice ingestion. However, it is apparent that consumption of large quantities of cranberry juice (about 1-2 L per day) or cranberry juice concentrates in supplements for an extended time period (>3-4 weeks) may temporally alter the effect of warfarin. Therefore, the total avoidance of cranberry juice by warfarin users may not be warranted by the published studies. However, in certain situations of higher intake of cranberry juice or concentrate there may be a need to monitor both warfarin doses and its effect.
\end{abstract}

This article is open to POST-PUBLICATION REVIEW. Registered readers (see "For Readers") may comment by clicking on ABSTRACT on the issue's contents page.

\section{INTRODUCTION}

The concept of health drinks has developed rapidly across the globe with the rapid emergence of several fruit juices with promising evidence for numerous therapeutic benefits. One such popular fruit beverage is cranberry juice, whose beneficial effects in preventing bacterial infections especially in urinary tract are well established (14). Cranberry juice has been extensively studied for numerous other therapeutic benefits ranging from diabetes, metabolic syndrome, obesity, prevention of risks of cardiovascular disease, helicopter pylori bacterial infections and dental diseases (5-9).

In today's treatment protocols of various comorbid medical conditions over-the-counter drugs and poly-pharmacy are commonly practised. Therefore, when multiple active drugs are ingested for risk prevention strategies there needs to be a vigil for any clinically relevant drug-drug interaction that may occur leading to either manifestation of an overt safety issue or compromising of efficacy resulting in a treatment failure (10-12). In this context, ingestion of fruit juices such as cranberry juice, especially in patient populations on poly-pharmacy, may introduce a further complication, since cranberry juice contains several active components (13) which may have the potential to interfere in the absorption, metabolism and/or excretion of the

Corresponding Author: Dr. Nuggehally R Srinivas, Suramus Biopharm, J.P. Nagar, I Phase, Bangalore 560078, India. Email: srini.suramus@yahoo.com 
interactions, especially the topic of grapefruit juice causing significant clinical herb-drug interactions, via CYP enzymes and transporters (14-17).

\section{SCOPE}

The intent of this review is to collate the in vitro, preclinical and clinical data of published work where potential interaction with cranberry juice has been evaluated and to provide perspectives in light of the evidence gathered on the potential unwanted effects of the use of cranberry juice from the point of view of clinical herb-drug interaction.

\section{METHODS}

Using a Medline (Pubmed) search with terms such as cranberry juice, pharmacokinetics, clinical, preclinical or in vitro, relevant articles pertaining to the published work on cranberry juice were screened using drug-drug interaction as a key search tool. Accordingly, data were collated into appropriate sub-headings pertaining to in vitro, preclinical and clinical studies for further data evaluation and interpretation.

\section{IN VITRO/PRECLINICAL}

\section{Human liver microsomal CYP3A - midazolam probe substrate}

Ngo et al (2009) have conducted an impressive investigation to pin-point the fraction of the cranberry juice that was more likely to inhibit human CYP3A4 enzyme (18). In this work, five different cranberry juice brands were tested in a blinded manner, since the active components present in the cranberry juice may vary based on the collection and/or storage of fruits and processing conditions (18). The incubation of midazolam as a probe for CYP3A4 in human liver microsomes was performed with the five cranberry juice brands and a control treatment. The formation of 1-hydroxymidazolam activity was monitored as the surrogate for CYP3A activity. Four of the five brands provided a concentration dependent inhibition of CYP3A activity in the human liver microsomes (18). However, the extent of inhibition at comparable time point varied between the four brands of cranberry juice. At the lowest amount of the juice tested $(0.05 \% \quad \mathrm{v} / \mathrm{v})$ the extent of CYP3A4 inhibition relative to the control was almost 2-fold different between the brand that produced the highest CYP3A4 inhibition (68\%) and one that had the least inhibition (34\%) (18). In only 2 out of 5 brands of cranberry juice tested at the higher concentrations $(0.5 \%, \mathrm{v} / \mathrm{v})$ was CYP3A4 activity completely inhibited, which was comparable to the complete inhibition produced by ketoconazole ( $2 \mu \mathrm{M}$ concentration) used as a positive control in this experiment (18). One brand of cranberry juice that produced maximum CYP3A inhibition was selected for further in vivo characterization. This brand of cranberry juice was subjected to extraction and fractionation into various solvent systems, namely hexane, chloroform, butanol and an aqueous soluble phase. Each of the four fractions was characterized for its ability to inhibit CYP3A4 activity using human liver microsomes and midazolam as the index substrate (18). All four fractions showed CYP3A inhibition in a concentration dependent manner. Interestingly, the CYP3A4 inhibition was strongly correlated with the hydrophobicity of the fraction. The more hydrophobic fraction (n-hexane) showed maximal CYP3A4 inhibition as compared to the least hydrophobic aqueous phase, relative to the control activity (18). Therefore, this crucial fractionation experiment provided a hint that the CYP3A inhibition activity of cranberry juice may be associated with more hydrophobic active constituents of the juice (18).

\section{Recombinant(r) CYPs (rCYP3A4 and rCYP3A5) - midazolam as probe substrate} Ngo et al (2009) evaluated the ability of the chosen brand of cranberry juice and the associated 4 fractions described above to inhibit the polymorphic CYP3A5 enzyme relative to CYP3A4 enzyme, using human recombinant enzymes and midazolam as the probe substrate and monitoring the formation of 1'hydroxymidazolam activity. This experiment was performed using both rCYP3A4 and rCYP3A5 systems with positive control, ketoconazole. It was evident that the intact juice and 4 fractions showed concentration-dependent inhibition of both recombinant CYP3A enzymes (18). However, rCYP3A4 inhibition was relatively higher as compared to rCYP3A5 inhibition across the 4 fractions and the intact cranberry juice. For instance, n-hexane fraction $(50 \mu \mathrm{g} / \mathrm{ml}$ concentration) inhibited rCYP3A4 and rCYP3A5 by $75 \%$ and $62 \%$, respectively. Regardless of the two rCYP3A enzymes tested, the n-hexane fraction showed the maximum inhibition compared to all other fractions (18). 


\section{Inhibition of absorptive permeability of midazolam across the small intestine}

Ngo et al (2009) utilized Caco-2 cell monolayers to test the role of cranberry juice on the absorptive permeability of an index substrate, midazolam. The absorption potential of midazolam across the monolayer was tested in different in vitro conditions that encompassed an acidic $\mathrm{pH}$ environment mimicking small intestine ( $\mathrm{pH}$ 5.5), diluted/pH neutralized $(\mathrm{pH} 7.4 ; 1: 4$ dilution of the juice) and high glucose concentration at neutral $\mathrm{pH}$ (glucose: $50 \mathrm{~g} / \mathrm{L}, \mathrm{pH}$ 7.4) (18). Since only one in vitro condition incorporated cranberry juice, the design could tease out if they were factors outside of cranberry juice, such as acidic conditions and/or high sugar content that would mediate the absorption of midazolam across the human intestine mono layers (18). The evidence suggested that only cranberry juice treatment had a significant effect on the transport of midazolam across the mono layers. The inhibitory effect on the absorption of midazolam was quite apparent after 1 hour of incubation. It was found that the amount of midazolam recovered in the basolateral compartment was at least 34 to $47 \%$ lower for the cranberry juice treatment as compared to control and other in vitro conditions tested. This neatly designed cascade of experiments unequivocally confirmed that cranberry juice had the potential to interfere in the permeability/transport of midazolam across the Caco 2-cell monolayers (18).

\section{Human liver microsomal CYP2C9 - warfarin as the probe substrate}

Ngo et al (2010) have evaluated the ability of cranberry juice to inhibit the human liver microsome CYP2C9 activity. In this work, five different cranberry juice brands were tested in a blinded manner at three concentration levels $(0.05 \%, 0.2 \%$ and $0.5 \% \mathrm{v} / \mathrm{v})$. Incubation of $\mathrm{S}$ warfarin as a probe for CYP2C9 in human liver microsomal preparations for 30 minutes was performed with the five cranberry juice brands and the control treatment (19). The formation of 7-hydroxywarfarin activity was monitored as the surrogate for CYP2C9 activity. Only one brand of cranberry juice out of 5 tested produced a concentration dependent inhibition of CYP2C9 activity in the human liver microsomes (19). It appeared that at a concentration of $0.2 \% \mathrm{v} / \mathrm{v}$ cranberry juice $>50 \%$ of CYP2C 9 activity was inhibited and at a higher concentration of $0.5 \%$ $\mathrm{v} / \mathrm{v}$ of cranberry juice almost $90-95 \%$ of CYP $2 \mathrm{C} 9$ activity was inhibited (19). The positive control sulfaphenazole inhibited the CYP2C9 activity by over $95 \%$, suggesting that at a higher concentration of $0.5 \% \mathrm{v} / \mathrm{v}$ or above cranberry juice was equally potent as a CYP2C9 inhibitor (19).

Each of the four fractions above was characterized on its ability to inhibit CYP2C9 activity in human liver microsomes using Swarfarin as the index substrate (19). All four fractions displayed CYP2C9 inhibition in a dose or concentration dependent manner. Interestingly, the CYP2C9 inhibition was strongly correlated with the chloroform fraction compared to either a more hydrophobic fraction (n-hexane) or more hydrophilic fraction (19). The chloroform fraction produced an inhibition of $32 \%$ and $75 \%$ at the tested concentrations of $10 \mu \mathrm{g} / \mathrm{ml}$ and $50 \mu \mathrm{g} / \mathrm{ml}$, respectively. Therefore, this crucial fractionation experiment provided a hint that the $\mathrm{CYP} 2 \mathrm{C} 9$ inhibition activity of cranberry juice may be associated with a different fraction of the juice as compared to CYP3A inhibition $(18,19)$.

\section{Human liver microsomal CYP2C9 - diclofenac as the probe substrate}

Ushigima et al (2009) evaluated the ability of cranberry juice to inhibit the human liver microsomal CYP2C9 activity using diclofenac as the probe substrate. The well-established potent CYP2C9 inhibitor sulfaphenazole was used as a positive control. Upon incubation with various amounts of cranberry juice, CYP2C9 activity in the human liver microsomes was inhibited in a concentration dependent manner, such that an $\mathrm{IC}_{50}$ value of $1.14 \% \mathrm{v} / \mathrm{v}$ was established for the cranberry juice (20). The relative $\mathrm{IC}_{50}$ value established for sulfaphenazole in the same human liver microsomes was $0.4 \mu \mathrm{M}$. This confirmed that cranberry juice at a higher dose was a potent inhibitor of CYP2C9, with complete abolition of the metabolism of diclofenac (20).

\section{Human liver microsomal CYP2C9 - flurbiprofen as the probe substrate}

Greenblatt et al (2006) demonstrated the ability of many beverages such as cranberry juice, grape juice and brewed tea to inhibit CYP2C9 mediated hydroxylation of flurbiprofen in a freshly prepared human microsomal system (21). Fluoconazole, a potent CYP2C9 inhibitor, was used as the positive control. Cranberry juice showed a concentration dependent inhibition of liver microsomal CYP2C9 and the $\mathrm{IC}_{50}$ value for the CYP2C9 inhibition was about $2.5 \% \mathrm{v} / \mathrm{v}(21)$. Interestingly, tea beverage showed much more 
potent CYP2C9 inhibition by a leftward shift in its response curve when superimposed on cranberry juice, with an $\mathrm{IC}_{50}$ value of about $1.2 \%$ for CYP2C9 inhibition (21). Grape juice also behaved in an identical manner to that of tea beverage (flurbiprofen hydroxylation was reduced by $10-11 \%$ of control at a concentration of $2.5 \%$ $\mathrm{v} / \mathrm{v})$. The index inhibitor sulfaphenazole inhibited flurbiprofen hydroxylation pathway by 4 -fold as compared to the control treatment $(21 \%)$ at 2.5 $\mu \mathrm{mol} / \mathrm{L}$. Fluconazole inhibited the CYP2C9 mediated activity of human liver microsomes with an $\mathrm{IC}_{50}$ value of $29.5 \mu \mathrm{M}(21)$. Therefore, it appeared that both tea beverage and grape juice had a greater potential of CYP2C9 inhibition than cranberry juice at equivalent concentrations of the three beverages (21).

\section{Rat intestinal microsomes/human liver} microsomes - nifedepine as the probe substrate The work of Ueswa and Mohri (2006) suggested that cranberry juice had the potential to inhibit the CYP3A mediated activity which was involved in the oxidation of nifedepine in both rat intestine microsomes and human liver microsomes (22). The decrease observed with cranberry juice pretreatment, however, was moderate (13-18\% inhibition) as compared to grapefruit juice pretreatment (58 - 63\% inhibition) in rat intestinal and human liver microsomal systems. It appeared that mechanism based inhibition of nifedipine oxidation by cranberry juice was not as potent as that observed with grapefruit juice (22).

\section{Preclinical - Nifedipine}

In an interesting study in rats, the pharmacokinetics of nifedipine was investigated with separate pre-treatment of cranberry juice and grapefruit juice in relation to water treatment (22). Each treatment group had 5 animals to support the statistical analysis of any observed pharmacokinetic differences (22). Both cranberry juice and grapefruit juice influenced the rate and extent of nifedipine oral absorption, relative to the control treatment. Although peak levels for nifedipine were numerically greater for both juice treatments (about 20\%) relative to water treatment this did not reach statistical significance (22). However, the $\mathrm{AUC}_{(0-\infty)}$ values for nifedipine were approximately 1.61 to 1.65 fold higher for both juice treatments as compare to the water comparator arm. With the exception of total body clearance of nifedipine, which was reduced by 40 $45 \%$ for both juice treatments relative to control, the other parameters such as volume of distribution, mean residence time and elimination half-life were not statistically significantly different from the control treatment values (22). Therefore, it was concluded that both cranberry juice and grapefruit juice reduced the presystemic clearance of nifedipine by inhibiting the intestinal CYP3A metabolism and improved the oral bioavailability of the drug. There was no evidence of inhibition of hepatic CYP3A metabolism of nifedipine by either of the two juices (22).

\section{CLINICAL DATA}

\section{Midazolam \\ Study 1}

Ngo et al (2009) evaluated the pharmacokinetics of midazolam in presence of a double-strength cranberry juice (double strength juice was prepared by reconstituting cranberry juice in half the amount of recommended water to yield a $200 \%$ concentrated juice) that produced the maximum CYP3A4 inhibition in the in vitro characterization of various brands of cranberry juice (18). The water treatment was used as the control arm. The study was a 2-way randomized crossover in 16 healthy volunteers with an adequate washout period between the treatments. Consistent with the in vitro cell permeability experiment, the presence of cranberry juice slowed the absorption rate of midazolam relative to the water treatment (18). Although there was variability, consistent lowering of $\mathrm{C}_{\max }$ of midazolam was observed, such that the geometric mean value for cranberry juice was at least $31 \%$ lower than the geometric mean obtained for the water treatment. The delay in time to peak levels of midazolam was evident in all subjects and a large variation of 2 to 8 -fold was observed (18). Although midazolam absorption rate was hindered by cranberry juice, the extent of absorption of midazolam was much higher for the cranberry juice treatment relatively to the water treatment. The geometric mean $\mathrm{AUC}_{(0-\infty)}$ value for midazolam was $33 \%$ higher for the cranberry juice relative to the control treatment (18). Furthermore, this increase in exposure was clearly reflected in the oral clearance of midazolam for cranberry juice, which was approximately $25 \%$ lower as compared to the water treatment (18). In spite of the observed changes in absorption rate and total exposure, the elimination half-life values for midazolam appeared to be consistent between the two treatments, suggesting that hepatic CYP system had no influence on the observed changes 
in midazolam pharmacokinetics with cranberry juice intake (18).

The measurement of the 1'hydroxymidazolam metabolite provided unequivocal confirmation that cranberry juice had an inhibitory effect on the intestinal CYP3A metabolism of midazolam (18). The formation and appearance of 1'-hydroxymidazolam in the systemic circulation was significantly delayed for the cranberry juice treatment and the geometric mean $\mathrm{C}_{\max }$ level for cranberry juice was almost 3fold lower than the water treatment (18). The extent of formation of metabolite measured by $\mathrm{AUC}_{(0-\infty)}$ value for 1'-hydroxymidazolam was much lower for the cranberry juice relative to water treatment, confirming that both rate and extent of metabolite formation was affected by cranberry juice due to the intestinal inhibition of CYP3A4 by cranberry juice. As was noted earlier for the parent compound, the elimination half-life for 1'-hydroxymidazolam was unaltered by cranberry juice, suggesting that cranberry juice had no impact on the hepatic enzymatic system (18). Interestingly, the computation of metabolite to parent $\mathrm{AUC}_{(0-\infty)}$ ratio for water treatment $(0.34)$ and cranberry juice $(0.21)$ also suggested that indeed bioavailability of midazolam increased, resulting in a greater exposure of the parent and lower formation of metabolite with cranberry juice intake (18).

\section{Study 2}

As part of a 3-drug cocktail probe, the pharmacokinetics of midazolam, used as a model substrate to measure the CYP3A4 activity, was studied after continuous pre-treatment with 200 $\mathrm{ml}$ of regular cranberry juice (regular cranberry juice was prepared by reconstituting cranberry juice with water in a 1:4 v/v) three times daily for 10 days relative to water control treatment (23). This was a randomized crossover study in 10 healthy volunteers, with two treatments (cranberry juice and water), and a long washout period that lasted for 4 weeks. On the study day (i.e., day 5), the frequency of intake of $200 \mathrm{ml}$ cranberry juice was changed to four times instead of three times. The pre-treatment with cranberry juice had no influence on the pharmacokinetic profile of midazolam as reflected by $\mathrm{AUC}_{(0-\infty)}$ values (cranberry juice: $4.32 \mathrm{ng} / \mathrm{ml} . \mathrm{hr}$ vs water: $4.38 \mathrm{ng} / \mathrm{ml} . \mathrm{hr}$ ), $\mathrm{C}_{\max }$ values (cranberry juice: 1.69 $\mathrm{ng} / \mathrm{ml}$ and water: $1.49 \mathrm{ng} / \mathrm{ml}$ ) and comparable elimination half-life values $(2.5-2.7 \mathrm{~h})$ between the two treatments $(23)$. The $\mathrm{AUC}_{(0-\infty)}$ ratio of midazolam/10-OH-midazolam closely matched the values between cranberry juice (4.26) and water treatments (4.35). In summary, under the experimental conditions studied cranberry juice (200 $\mathrm{ml}$ given three times daily and then four times daily for 10 days) did not inhibit CYP3A4 activity, as evidenced by similar pharmacokinetic data of the parent midazolam and its metabolite (23).

\section{Warfarin \\ Study 1}

The study involved the use of a double strength cranberry juice (a total of $720 \mathrm{ml}$ of cranberry juice was consumed in three- $240 \mathrm{ml}$ aliquots, with each $240 \mathrm{ml}$ aliquot of cranberry juice consumed in a 15-min time interval) that had shown the highest in vitro inhibition of CYP2C9 in human liver microsomes as measured by the 7hydroxylation pathway of S-warfarin (19). In total, 16 volunteers participated in this study which was a crossover design of two treatments, double-strength cranberry juice and the water control arm. Cranberry juice had a measurable impact by slowing the absorption rate of both warfarin enantiomers, as reflected in the lowering of peak concentration levels (19). There was a relatively long delay in the time for the appearance of the peak levels in plasma (2-fold and 3-fold for R-warfarin and S-Warfarin, respectively). The geometric mean of $\mathrm{C}_{\max }$ for $\mathrm{S}$ warfarin $(1.5 \mu \mathrm{M})$ for the cranberry juice treatment was $25 \%$ lower than that of the water treatment $(2 \mu \mathrm{M})$. Similarly, the mean $\mathrm{C}_{\max }$ for Rwarfarin $(1.4 \mu \mathrm{M})$ for the cranberry juice treatment was $>20 \%$ lower than water treatment $(1.7 \mu \mathrm{M})$ (19). Hence, for both warfarin enantiomers the $90 \%$ confidence interval for bioequivalence between the cranberry juice and water treatments was outside the $80-125 \%$ window, suggesting that peak concentrations were significantly affected by the cranberry juice (19). In contrast, the $\mathrm{AUC}_{(0-\infty)}$ values tended to be similar for the cranberry juice treatment relative to water treatment for both warfarin enantiomers. Hence the comparison of the geometric point estimate with $90 \%$ confidence intervals rendered bioequivalence for either of the two warfarin enantiomers between the cranberry juice and water treatments (19). The elimination half-life values appeared to be similar between the two treatments for both R- and S-warfarin. Overall, although it appeared that cranberry juice may have altered the absorption rate of both warfarin enantiomers, it did not significantly alter the 
extent of absorption of the two warfarin enantiomers, relative to the control treatment (19).

\section{Study 2}

As part of a 3-drug cocktail probe the pharmacokinetics of warfarin, an index substrate to measure the CYP2C9 activity, was studied after continuous pre-treatment with $200 \mathrm{ml}$ of regular cranberry juice (regular cranberry juice was prepared by reconstituting cranberry juice with water in a $1: 4 \mathrm{v} / \mathrm{v}$ ) three times daily for 10 days relative to water control treatment (23). This was a randomized crossover study in 10 healthy volunteers, with two treatments (cranberry juice and water) and a long washout period that lasted 4 weeks. On the study day (i.e. day 5), the frequency of intake of $200 \mathrm{ml}$ cranberry juice was changed to four times daily instead of three times. The pre-treatment with cranberry juice showed no alterations in the pharmacokinetic parameters of either warfarin enantiomers (23). The $\mathrm{AUC}_{(0-\infty)}$ values (cranberry juice: R-warfarin $26.8 \mathrm{ng} / \mathrm{ml} . \mathrm{hr}$; S-warfarin $19.3 \mathrm{ng} / \mathrm{ml} . \mathrm{hr}$ vs water: R-warfarin $26.4 \mathrm{ng} / \mathrm{ml} . \mathrm{hr}$; S-warfarin $20.7 \mathrm{ng} / \mathrm{ml} . \mathrm{hr})$ ), $\mathrm{C}_{\max }$ values (cranberry juice: R-warfarin $0.49 \mathrm{ng} / \mathrm{ml} . \mathrm{hr}$; S-warfarin $0.50 \mathrm{ng} / \mathrm{ml}$ vs water: R-warfarin 0.51 $\mathrm{ng} / \mathrm{ml} . \mathrm{hr} ; \quad$ S-warfarin $0.52 \mathrm{ng} / \mathrm{ml} . \mathrm{hr})$ ), and elimination half-life values (cranberry juice: Rwarfarin $44.7 \mathrm{hr}$; S-warfarin $36.4 \mathrm{hr}$ vs water: Rwarfarin $44.4 \mathrm{hr}$; S-warfarin $40.3 \mathrm{hr}$ ) were found to be similar between the two treatments (23). The pharmacodynamic variables such as

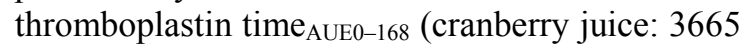
s $\mathrm{hr}$ vs water: $3624 \mathrm{~s} \mathrm{hr}$ ) and $\mathrm{E}_{\max }$ for thromboplastin time (cranberry juice: $24.5 \mathrm{~s}$ vs water: $24 \mathrm{~s}$ ) measured in this study were found to be similar for the two treatments, suggesting that cranberry juice did not impair the anti-coagulation activity of warfarin (23). In summary, under a rigorous daily dosing of cranberry juice, there were no apparent signs of inhibition CYP2C9 activity, as evidenced by similar pharmacokinetic data and pharmacodynamics of warfarin enantiomers between the two treatments (23).

\section{Study 3}

Ansell et al (2009) studied the pharmacokineticpharmacodynamic interaction of warfarin with cranberry juice in a blinded manner in 30 patients (14 patients assigned to cranberry juice and 16 patients assigned to water control treatment) (24). The chosen patients for this study were on a stable dose of warfarin to achieve the required therapeutic anti-coagulation level, as determined by the INR. The 30 patients per the randomization schedule either received cranberry juice (approximately $240 \mathrm{ml}$ concentrate containing $27 \%$ cranberry juice) or the matching placebo treatment (approximately $240 \mathrm{ml}$ of placebo similar in colour and taste with that of cranberry juice concentrate) once daily for 14 days (24). The plasma concentrations of warfarin enantiomers were determined during the dosing period of 2 weeks and one-week follow up thereafter. Similarly, INR values were noted to measure the effect of cranberry juice on the dynamics of warfarin (Ansell et al, 2009) (24). No statistically significant differences were noted in the plasma concentrations of either S-warfarin or R-warfarin when appropriate comparisons (leadin period or treatment period) were made between the cranberry juice vs placebo treated groups (24). The pharmacokinetic results confirmed the earlier reports that cranberry juice may not inhibit warfarin metabolism mediated via CYP2C9. In terms of pharmacodynamics, the pre-treatment with cranberry juice did not have a significant impact on the measured INR values at various time points during the lead-in, treatment or follow-up period of the investigation, with the exception of a single point on day 12 of treatment. Although numerically INR values appeared to be small, differences were statistically significant compared to placebo treatment (24).

\section{Study 4}

Mellen et al (2009) carried out a prospective pharmacodynamic interaction study in patient populations which were stabilized for a therapeutic anti-coagulation effect of warfarin with INRs of 2-3 (25). No change in warfarin dose for a 4 week observation period and a maximum of $10 \%$ fluctuation in the repeat INR measurements were key inclusion criteria for patient enrolment in this prospective study (25). There were ten patients that enrolled in this study. The cranberry juice was ingested every day ( 240 $\mathrm{ml}$ juice on a twice daily schedule) for 7 days by all patients. The patients continued on their daily dose of warfarin during the entire study. Both INRs and prothrombin times were measured at day 0 prior to cranberry juice intake and on days 2, 6 and 8 of the study (25). The results confirmed that cranberry juice had no apparent effect on prothrombin times on days 2,6 and 8 when compared to the baseline value collected on day 0 (it appeared to be within the defined boundary of 12.9 to 15$)$. In addition, the pre-treatment with double strength juice had no impact on INRs, which remained stable between 2 and 3 during the 
entire study protocol (25). The data from one patient was excluded since the patient developed diarrhoea soon after study initiation and this patient did not consume any more cranberry juice and his warfarin dose was withheld until the diarrhoea resolved (25).

\section{Study 5}

Abdul et al (2008) reported a pharmacodynamic interaction between cranberry juice and a single high dose of warfarin in healthy subjects (26). In a three-way randomized crossover design, 12 healthy male subjects received one of three treatments: a) single dose of warfarin after 2-week pre-treatment with cranberry juice concentrate capsules (1000 mg cranberry juice concentrate, three times daily) followed by another week of cranberry juice concentrate capsules; b) single dose of warfarin 2-week pre-treatment with garlic and c) single dose of warfarin with water (26). The PKPD assessments were done by quantifying the plasma concentrations of R- and S-warfarin and the measurement of individual subject INR. Cranberry juice concentrate or garlic did not alter the pharmacokinetics of either of the two enantiomers relative to the control treatment and produced identical exposure and elimination halflife values (26). However, cranberry juice concentrate but not garlic showed statistically significant difference in the INR value - almost $30 \%$ increases in the area under the INR time curve- suggesting that there was a potential susceptibility for a pharmacodynamic interaction in the patient population (26). Another important finding of this study was that both cranberry juice concentrate and garlic provided hints of VKORC1 (not CYP2C9) genotype-dependent interactions with warfarin, which may have bearing in the patient population (26).

\section{Study 6}

$\mathrm{Li}$ et al (2006) performed a blinded 2-way crossover study that involved comparison of pretreatment of cranberry juice vs water for 7 days in 7 patients with atrial fibrillation on a stable dose of warfarin for at least 3 months (27). The washout period was for a week between the two treatments. The pharmacodynamic measurements included the computation of INR values on various days during the treatment period (27). The INR during treatment days were not significantly different from the baseline INR values obtained prior to study initiation for either cranberry juice or water treatments. For example, the INR values for cranberry juice and water on day 7 were 2.23 and 2.16, respectively, as compared to the corresponding baseline values of 2.28 and 2.13, respectively (27). It was concluded that avoidance of cranberry juice was not necessary for patients on warfarin; however, a close vigil on INR was recommended (27).

\section{Diclofenac}

The clinical study was performed as a 2-way randomized crossover with cranberry juice (180 $\mathrm{ml}$ of $27 \%$ cranberry juice concentrate, twice daily for 5 days $)$ and water $(180 \mathrm{ml}$ of water, twice daily for 5 days) treatments with at least a week wash-out period (20). The study involved 8 subjects who had similar CYP2C9 genotype status $\left({ }^{*} 1 / * 1\right)$ to ensure that the outcome was not influenced by the genetic composition of the subjects (20). Following a single oral dose of diclofenac, there appeared to be no alteration in either the rate or extent of absorption of diclofenac in cranberry juice treatment relative to the water control. A similar trend was observed in the absorption and disposition of diclofenac upon multiple daily doses of cranberry juice, suggesting that the in vitro observations of CYP2C9 inhibition could not be replicated in the clinic (20). Since the protein bound and unbound fraction of diclofenac was measured in this study for both in vivo plasma samples and in the in vitro liver microsomes, there was a hint that the protein binding of diclofenac may have played a role to explain the discrepancy between the in vitro and in vivo findings. Because a major part of diclofenac existed in the unbound form in vitro it enabled a constant delivery of the substrate to fuel the enzymatic activity; however, in vivo the high protein binding of diclofenac may have limited the supply of the substrate to the metabolising enzyme (20).

\section{Amoxicillin}

Li et al (2009) investigated the pharmacokinetics of amoxicillin after a chronic dosing with cranberry juice (chronic dosing of cranberry juice comprised of administration of approximately 360 $\mathrm{ml}$ of cranberry juice, twice daily, for two days) relative to a water control treatment (28). The study was conducted in eighteen healthy female subjects with four treatments administered in a fixed-sequence crossover manner with a 7-day washout period. Two doses of amoxicillin (500 $\mathrm{mg}$ and $2 \mathrm{~g}$ ) were used with and without $180 \mathrm{ml}$ of co-administered cranberry juice at the time of dosing (28) With the exception of a shift in time to peak levels of amoxicillin (approximately $1 \mathrm{hr}$ ), 
cranberry juice did not have any other impact on the pharmacokinetic disposition of amoxicillin at both doses tested relative to water control treatment. For example at the $500 \mathrm{mg}$ dose, the AUC (cranberry juice: $23.7 \mu \mathrm{g} / \mathrm{ml} . \mathrm{hr}$ vs water: $24.9 \mu \mathrm{g} / \mathrm{ml} . \mathrm{hr})$, oral clearance ((cranberry juice: $367 \mathrm{ml} / \mathrm{min}$ vs water: $347 \mathrm{ml} / \mathrm{min})$, elimination half-life ((cranberry juice: $1.05 \mathrm{~h}$ vs water: $1.13 \mathrm{~h})$ and renal clearance (cranberry juice: $236 \mathrm{ml} / \mathrm{min}$ vs water: $218 \mathrm{ml} / \mathrm{min}$ ) were almost identical for both treatments (28). Similarly, cranberry juice had negligible influence on the derived pharmacokinetic parameters at the $2 \mathrm{~g}$ dose of amoxicillin. The results with amoxicillin appeared to suggest that cranberry juice had a limited role in the inhibition of efflux transporters that would impede oral drug transport/permeability of certain categories of drugs (28).

\section{Ceflacor}

$\mathrm{Li}$ et al (2009) have reported the clinical pharmacokinetics of ceflacor after a chronic dosing with cranberry juice (chronic dosing of cranberry juice comprised of administration of approximately $360 \mathrm{ml}$ of cranberry juice, twice daily, for two days) relative to a water control treatment (28). The study was conducted in eighteen healthy female subjects in a randomised crossover fashion with a 7-day washout period between the two treatments. A single dose of 500 mg ceflacor was administered in this study with and without $180 \mathrm{ml}$ of co-administered cranberry juice at the time of dosing. Cranberry juice did not have any influence on the pharmacokinetic parameters of ceflacor relative to water control treatment (28). For example, the AUC (cranberry juice: $20.5 \mu \mathrm{g} / \mathrm{ml}$.hr vs water: $21.2 \mu \mathrm{g} / \mathrm{ml} . \mathrm{hr}$ ), oral clearance ((cranberry juice: $408 \mathrm{ml} / \mathrm{min}$ vs water: $397 \mathrm{ml} / \mathrm{min}$ ), elimination half-life ((cranberry juice: $0.62 \mathrm{hr}$ vs water: $0.59 \mathrm{hr}$ ) and renal clearance (cranberry juice: $310 \mathrm{ml} / \mathrm{min}$ vs water: $307 \mathrm{ml} / \mathrm{min}$ ) were almost overlapping between the two treatments (28).

\section{Tizanidine}

As part of a 3-drug cocktail probe the pharmacokinetics of tizanidine, a model substrate to measure the CYP1A2 activity, was studied after continuous pre-treatment with regular cranberry juice three times daily for 10 days relative to water control treatment (23). This was a randomized crossover study in 10 healthy volunteers, with two treatments (cranberry juice and water) and a long washout period that lasted 4 weeks (23). On the study day (i.e. day 5), the frequency of intake of $200 \mathrm{ml}$ cranberry juice was changed to four times instead of three times. The pharmacokinetic disposition of tizadine was unaltered by the pre-treatment of cranberry juice as gauged by $\mathrm{AUC}_{(0-\infty)}$ values (cranberry juice: $2.16 \mathrm{ng} / \mathrm{ml}$.hr vs water: $2.58 \mathrm{ng} / \mathrm{ml}$.hr) and almost similar elimination half-life values $(1.2-1.3 \mathrm{hr})$ between the two treatments. Two metabolites of tizadine (M-3 and M-4) were also quantitated and the $\mathrm{AUC}_{(0-\infty)}$ ratio of tizadine/M-3 was identical between cranberry juice (5.13) and water treatments (4.86) (23). Similarly, $\mathrm{AUC}_{(0-\infty)}$ ratio tiazidine/M-4 was comparable between cranberry juice (5.89) and water treatments (6.43). In summary, cranberry juice did not inhibit CYP1A2 activity, as evidenced by the unaltered data of the parent tizanidine and its two major metabolites, M-3 and M-4 (23).

\section{Flurbiprofen}

The investigation reported by Greenblatt et al (2006) evaluated the effect of pre-treatment of several beverages such as cranberry juice, grape juice and tea on the pharmacokinetics of flurbiprofen relative to water control treatment (21). This was a randomized design in fourteen healthy volunteers that had five treatment arms, namely: water/placebo, cranberry juice, tea beverage, grape juice and fluconazole, with one week washout period (21). The beverage treatment was applied the night before and 30 minutes (on each of the two occasions, approximately $240 \mathrm{ml}$ of the respective beverage was consumed) before flurbiprofen administration on the study day. The pharmacokinetics of flurbiprofen was unaffected by the pre-treatment with cranberry juice, as indicated by the extent of absorption, $\mathrm{AUC}_{(0-\infty)}$ (cranberry juice: $62 \mathrm{ug} / \mathrm{ml} . \mathrm{hr}$ vs placebo: 59 ug/ml.hr), oral clearance (cranberry juice: 29.3 $\mathrm{ml} / \mathrm{min}$ vs placebo: $30.5 \mathrm{ml} / \mathrm{min}$ ) and elimination half-life values (cranberry juice: $3.4 \mathrm{~h}$ vs placebo: 3.3 h) (21). Also, Cranberry juice produced identical exposure of 4-hydroxyflurbiprofen (1.16 $\mathrm{ug} / \mathrm{ml} . \mathrm{hr}$ ) as compared to placebo treatment (1.24 $\mathrm{ug} / \mathrm{ml} . \mathrm{hr}$ ) (21). In sharp contrast, fluconazole pretreatment, increased the exposure of flurbiprofen by almost 2-fold relative to placebo and decreased the oral clearance of flurbiprofen by an identical 2-fold margin as compared to placebo. The potent CYP2C9 inhibition of flurbiprofen also resulted in an almost 55\% reduced exposure of 4-hydroxyflurbiprofen relative to placebo treatment (21). The other beverages (grape juice and brewed tea) failed to 
interfere with the pharmacokinetics and metabolism of flurbiprofen under in vivo conditions, contrary to the in vitro CYP2C9 inhibition data (21).

\section{Cyclosporine}

The work of Grenier et al (2006) evaluated the influence of the co-administered fruit juices such as cranberry juice $(240 \mathrm{ml})$ and pomelo juice $(240$ $\mathrm{ml}$ ) on the pharmacokinetics of cyclosporine relative to $240 \mathrm{ml}$ water treatment (29). This was a 3-way randomized crossover design, with three sequences and 3 treatments with a 2-week washout period involving 12 healthy human volunteers. Pomelo juice but not cranberry juice showed significant impact on the pharmacokinetics of orally administered cyclosporine (29). Both absorption rate and extent of absorption of cyclosporine were higher following pre-treatment with pomelo juice $\left(\mathrm{C}_{\max }\right.$ values: pomelo juice: $1494 \mathrm{ng} / \mathrm{ml}$ vs cranberry juice: 1262 vs water: $1327 \mathrm{ng} / \mathrm{ml}$ and $\mathrm{AUC}_{(0-\infty)}$ values: pomelo juice: $6835 \mathrm{ng} / \mathrm{ml} . \mathrm{hr}$ vs cranberry juice: $5217 \mathrm{ng} / \mathrm{ml} . \mathrm{hr}$ vs water: $5753 \mathrm{ng} / \mathrm{ml} . \mathrm{hr}$ ) relative to other two treatments (29). However, the elimination half-life of cyclosporine (10-11 hr) was comparable across all treatments, suggesting that the effect of pomelo juice was only on the intestinal metabolism/transport of cyclosporine (29).

\section{Salicylate}

The work of Duthie et al (2005) confirmed the positive effect of cranberry juice intake on the intestinal absorption of endogenous salicylic acid. The study consisted of parallel treatments of cranberry juice $(250 \mathrm{~mL}$ three time daily) and placebo $(250 \mathrm{~mL}$ water three times daily) for 2weeks in two cohorts of healthy female subjects $(\mathrm{n}=11$ per group) who were matched for demographics such as height, weight and age (30). Assays were set up to quantify the levels of salicylates (salicylic acid and salicyluric acid) in urine and salicylic acid in plasma. The cohort that had daily intake of cranberry juice produced higher levels of salicylates in the urine within the first week of treatment and by the second week of treatment the plasma salicylic acid concentrations were higher than the placebo group (30). The study confirmed that regular intake of cranberry juice would significantly improve the absorption of endogenous salicylates for potential therapeutic anti-inflammatory benefit (30).

\section{DISCUSSION}

\section{General}

The evidence presented in this review, assimilating the extensive research on cranberry juice for over a decade, has suggested that cranberry juice has the potential to inhibit enzymes (CYP3A and CYP2C9) and transporter (Pgp) under in vitro conditions (Table 1). The in vitro potency of CYP3A inhibition for cranberry juice appeared to be similar or superior to grapefruit juice and pomegranate juice [Figure 1]; however, cranberry juice may not be as potent as grapefruit juice, tea beverage or pomegranate juice in its in vitro inhibition of CYP2C9 [Figure 2]. Nevertheless, the $\mathrm{IC}_{50}$ values attained in these studies suggested that a $240 \mathrm{ml}$ volume of regular cranberry juice or double-strength cranberry juice would contain reasonable amounts of the active principles to translate the observed in vitro findings into a clinically relevant interaction in humans. However, in all the clinical case studies gathered in this review from a pharmacokinetic perspective, with the exception of a single report of a mild-moderate CYP3A4 inhibition of the probe substrate midazolam, none of the substrates whose oxidative metabolism was dependent on several CYP enzymes (CYP34, CYP2C9 or CYP1A2) or transporters (Pgp) showed clinically relevant pharmacokinetic interaction upon pretreatment with cranberry juice (Table 1).

\section{Bioavailability of anthocyanins from cranberry juice}

The minimal in vivo translatability of the observed in vitro CYP inhibition leads to the key question of whether or not the active principles from cranberry juice responsible for drug interactions are absorbed from the gut into the systemic circulation. No or limited absorption of relevant cranberry juice components would translate into the lack of in vivo effects on the pharmacokinetics of the probe substrates. The interesting work of Milbury et al (2010) performed in 15 healthy subjects suggested that several anthocyanins were bioavailable following the acute ingestion of cranberry juice (31). Out of 15 active anthocyanins present in cranberry juice, at least 7 anthocyanins were measurable in plasma for up to 4 hours post the acute ingestion of cranberry juice. Despite high inter-subject variability, exposure and pharmacokinetics were defined for several active principles in this study (31). The highest circulating species was peonidin 3-galactoside (AUC: $18.7 \mathrm{nmol} . \mathrm{h} / \mathrm{L}$ ) and the 
remaining circulatory species were in the following rank order: cyanidin 3-arabionside, cyanidin 3-galactoside, peonidin 3-arabinoside, peonidin 3-glucoside, cyanidin 3-glucoside and malvadin 3-glucoside with AUC values of 13.5, $6.89, \quad 5.35,3.72, \quad 3.66$ and $2.55 \mathrm{nmol} . \mathrm{h} / \mathrm{L}$, respectively (31). The urinary recoveries of the active principles were very low and ranged from $0.007 \%$ to $0.03 \%$ of the administered dose. Because the study objective was to explore if anthocyanin concentrations levels reach a threshold required to elicit in vivo quenching of reactive oxygen species, it was concluded that concentrations attained in plasma were low and possibly insufficient to have a direct role (31). However, it was speculated that the plasma levels may potentially induce signal transduction and gene expression pathways. An extrapolation of the observations from this study to the context of lack of in vivo CYP inhibition by cranberry juice would lead to the speculation that concentrations of the active principles were potentially too low for a clinically relevant drug-drug interaction (31).

\section{CYP3A4 inhibition - human relevancy}

The in vitro inhibition of CYP3A and as well mechanism based CYP3A inhibition, similar to grapefruit juice, would put cranberry juice in the same league of grapefruit juice for potential CYP3A mediated drug interactions. Furthermore, the unequivocal in vivo inhibition of nifedipine metabolism in rats and overwhelming increase in bioavailability of nifedipine, almost comparable to that of grapefruit juice, suggested that perhaps it may be a strong CYP3A4 inhibitor in humans (22). However, the two clinical studies performed using midazolam as the substrate had disparate findings $(18,23)$. While one study showed no change in the bioavailability of midazolam (23), the other study showed a modest increase in the bioavailability of midazolam (18).

Although it is difficult to pin-point the cause for the discrepancy between the two studies, it is possible that the in vivo absorption of anthocyanins from the cranberry juice administration may have differed widely between the two studies, since one study presented a large single acute intake of cranberry juice whilst the other studyhad a chronic intake of cranberry juice (intake of 3 volumes of $240 \mathrm{~mL}$ double strength cranberry juice vs three times daily intake of 200
$\mathrm{mL}$ cranberry juice for 5 days $)(18,23)$. To underscore this view, a recent study on the oral bioavailability of active principles of cranberry juice suggested high variability and low systemic bioavailability of only 7 of the active anthocyanins (31). Therefore, it is conceivable that a daily intake or perhaps even a twice daily intake of cranberry juice may not produce enough circulatory anthocyanins to show inhibition of human CYP3A4, which was evident from another clinical study of a second CYP3A4 substrate, cyclosporine, which showed no change in the bioavailability and pharmacokinetics of cyclosporine from cranberry juice pre-treated subjects (29). In sharp contrast and to put things into perspective, pre-treatment of grapefruit juice consistently increased the bioavailability of both midazolam (AUC change: $52 \%$ to $106 \%)(32,33)$ and cyclosporine (AUC change: $45 \%$ to $60 \%$; 34 36)) in human subjects. These data suggested that cranberry juice, unlike grapefruit juice, would not cause a clinically relevant drug-drug interaction at the dose concentrations studied.

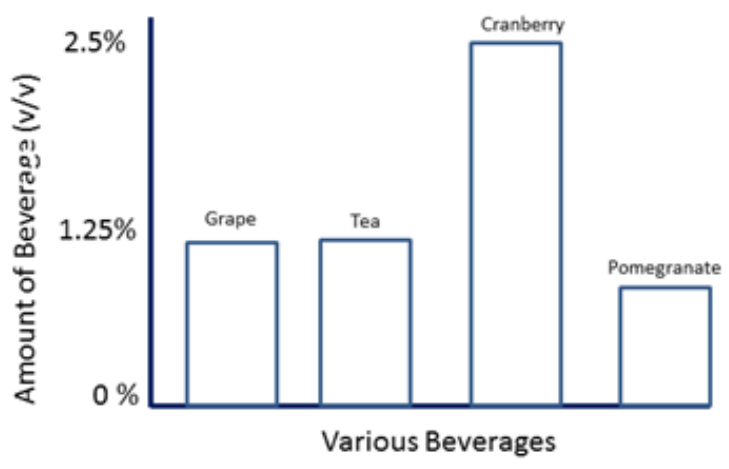

Figure 1. Schematic representation of CYP3A4 inhibition by various juice $-\mathrm{IC}_{50}$ values. The schematic illustration is to provide a quick visual basis for the likelihood of any potential in vivo pharmacokinetic interaction following the consumption of various beverages at the provided threshold levels. The work of Hidaki et al (Ref \# 44) showed that grapefruit juice and pomegranate juice was almost equi-potent in the inhibition of CYP3A4 mediated hydroxylation of carbamazepine in human liver microsomes. The data of cranberry juice was obtained from another study (Ref \#19) and is represented for comparative purpose only because midazolam was used as the probe substrate. 
Table 1. In vitro vs in vivo comparison to assess drug-drug interaction potential in the pharmacokinetics of cranberry juice.

\begin{tabular}{|c|c|c|c|}
\hline \multicolumn{2}{|c|}{ Absorption } & \multicolumn{2}{|c|}{ Metabolism } \\
\hline In vitro & In vivo & In vitro & In vivo \\
\hline $\begin{array}{l}\text { Inhibition of } \\
\text { Pgp transport of } \\
\text { midazolam }\end{array}$ & $\begin{array}{l}\text { No impact on } \\
\text { bioavailability or } \\
\text { disposition of either } \\
\text { amoxicillin or ceflacor }\end{array}$ & $\begin{array}{l}\text { CTP3A inhibition (midazolam, } \\
\text { nifedipine) rCYP3A4 / rCYP3AS } \\
\text { inhibition (midazolam) }\end{array}$ & $\begin{array}{l}\text { Midazolam ( } 2 \text { studies): modest } \\
\text { bioavailability increase }(25 \%) \text { in } \\
\text { one study but no change in the } \\
\text { second Cyclosporine: no change } \\
\text { in bioavailability or } \\
\text { pharmacokinetics } \\
\text { Nifedipine: increase } \\
\text { bioavailability ( } 65 \%) \text { in rats (no } \\
\text { human data available) }\end{array}$ \\
\hline & & $\begin{array}{l}\text { CYP2C9 inhibition (warfarin, } \\
\text { diclonfenac, flurbiprofen) }\end{array}$ & $\begin{array}{l}\text { Warfarin ( } 5 \text { studies): no charge } \\
\text { in the oral bioavailability or } \\
\text { pharmacokinetics }\end{array}$ \\
\hline & & & $\begin{array}{l}\text { Diclofenac: no alteration of } \\
\text { bioavailability or } \\
\text { pharmacokinetics }\end{array}$ \\
\hline & & & $\begin{array}{l}\text { Flurbiprofen: no alteration of } \\
\text { bioavailability or } \\
\text { pharmacokinetics }\end{array}$ \\
\hline & & CYP1A2 inhibition (unknown) & $\begin{array}{l}\text { Tizanidine: pharmacokinetics } \\
\text { and oral bioavailability was not } \\
\text { altered }\end{array}$ \\
\hline
\end{tabular}

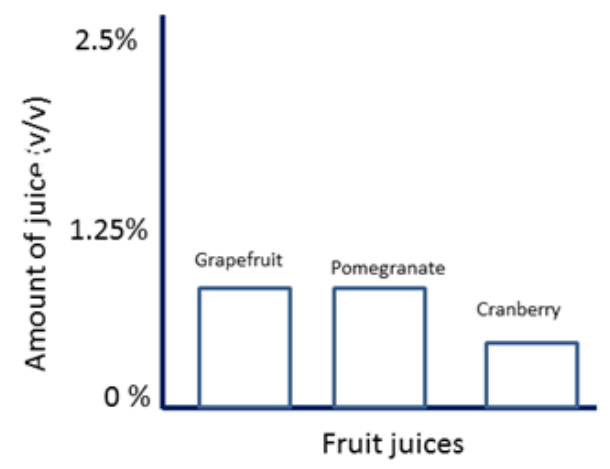

Figure 2. Schematic representation of CYP2C9 inhibition by various juice $-\mathrm{IC}_{50}$ values. The schematic illustration is to provide a quick visual basis to anticipate the likelihood of any potential in vivo pharmacokinetic interaction following the consumption of various beverages at the provided threshold levels. The work of Greenblatt et al (Ref \# 21) showed that cranberry juice was not as potent as either grape juice or tea beverage in the inhibition of CYP2C9 mediated hydroxylation of flurbiprofen in human liver microsomes. The data of pomegranate juice was obtained from another study (Ref \# 45) and is represented for comparative purpose only because diclofenac was used as the probe substrate.

To further underscore this view, the recently published work of Kim et al (2011) suggested the presence of three triterpines (masilinic acid, corosolic acid and ursolic acid) that caused inhibition of CYP3A in the human intestine microsomes and rCYP3A4 enzyme. However, relatively higher $\mathrm{IC}_{50}$ concentrations were required for the in vitro inhibition: 7.4, 8.8 and $<10 \mu \mathrm{M}$ (human intestine microsomes) and 2.8, 4.3 , and $<10 \mu \mathrm{M}$ (rCYP3A4), for masilinic acid, corosolic acid and ursolic acid, respectively (37). It is quite probable that under in vivo conditions the triterpene levels may not have reached the threshold levels for any significant inhibition of either intestinal or hepatic CYP3A4 enzymes in humans (37).

\section{CYP2C9 inhibition - Human relevancy Pharmacokinetics}

The in vitro evidence of cranberry juice as a potent CYP2C9 inhibitor was demonstrated for several substrates such as flurbiprofen, diclofenac and warfarin [Table 1]. At higher amounts, cranberry juice was as potent as fluconazole, which is a strong CYP2C9 inhibitor under in vitro and in vivo conditions. However, review of all the clinical data involving cranberry juice provided no evidence of altered bioavailability or pharmacokinetics of several substrates such as warfarin, diclofenac and fluribprofen whose metabolism was mediated by CYP2C9 (19-21, 23). Therefore, lack of in vivo inhibitory effects of cranberry juice suggested that under in vivo conditions either the active anthocyanin principles were not reaching adequate levels to produce any 
CYP2C9 inhibition or the intake amount of cranberry juice itself was insufficient. Since the cranberry juice intake differed (regular vs doublestrength; whole juice vs diluted concentrate etc), as well as the sourcing of the juice itself in the described studies, it may be difficult to pin-point an exact reason for the lack of in vivo inhibition. A number of speculative reasons could be put forward to explain the discrepancy: a) under in vitro conditions all 15 anthocyanin principles may be available to have a concerted effort in CYP2C9 inhibition; however, only 6 or 7 anthocyanin principles were bioavailable and at varying low levels in an in vivo study; b) a faster clearance of the active anthocyanin principles under in vivo conditions may have led to levels not reaching the threshold level to show CYP2C9 inhibition; c) influence of protein binding and/or rapid tissue uptake of the substrate would have been relevant under in vivo conditions, thereby preventing the drug availability to the enzymes. Overall, the clinical data clearly suggested that with daily or chronic intake of normal amounts of cranberry juice there is minimal or negligible potential to show a clinical relevant drug-drug interaction via CYP2C9 pathways.

\section{Pharmacodynamics}

The pharmacodynamics aspects of drug-drug interaction potential of cranberry juice in patients prescribed warfarin have received considerable attention. As evident in Table 2, there was one instance of elevated INR in healthy subjects after a single high dose of warfarin following ingestion of a cranberry juice concentrate supplement (26) and an isolated incidence of an increased INR at one particular time point following ingestion of juice during a 2-week treatment period in patients who were stabilized on warfarin (24). It could be argued that the observation of a small elevated INR in healthy subjects may not have relevance to a patient population and the episode of increased INR in patients at a single time point during multiple points of evaluation could be due to a random occurrence without clinical consequence. Other prospective studies performed with adequate controls and rigor had concluded that pre-treatment of cranberry juice had a negligible influence on the pharmacodynamics of warfarin $(21,25,27)$. Interestingly, these clinical studies $(21,25,27)$ used cranberry juice, unlike the Abdul et al (2008) study which used cranberry juice concentrate (26), raising speculation on the likely differences in the distribution of active principles between a cranberry concentrate vs juice. In this context, it is important to point out that several case reports have been published in the past several years that claim that ingestion of cranberry juice effects the INR and de-stabilizes the warfarin dose, necessitating a dose readjustment to obtain therapeutic anti-coagulant effect (38-42). In most of the instances, the acute consumption of cranberry juice was not deemed very high, with the exception of one or two instances where the patient drank almost $750 \mathrm{~mL}$ to $2 \mathrm{~L}$ of cranberry juice per day. In these patients a temporal relationship with the observed effect may be possible, since the study of Abdul et al (2008) reported mild INR alterations in healthy subjects with a larger cranberry dose consumption ( 2 capsules of $500 \mathrm{mg}$ cranberry juice concentrate given 3 times daily) and longer duration (3-weeks in total) relative to other prospective clinical studies (26). While there is circumstantial evidence that patients' noncompliance to follow dosing/study instructions (i.e. excess consumption of cranberry juice or concentrate) can lead to aberration in warfarin pharmacodynamics, there could be numerous other factors that may contribute to elevated INR values, such as co-morbid conditions, severe diarrhoea, concomitant drug use, genetic predisposition etc. Nevertheless, when all the findings are put in perspective, the debate whether or not cranberry juice affects pharmacodynamics of warfarin still continues to be unresolved. However, the view of total avoidance of cranberry juice by warfarin users does not appear to be justified since moderate consumption of cranberry juice per se in most studies appear not to affect the pharmacodynamics of warfarin. To underscore this point, Zikria et al (2010) have critically reviewed a number of prospective clinical studies and case reports pertaining to cranberry juice - warfarin interaction and concluded that the warning label of interaction potential between warfarin and cranberry juice needs to be re-visited by the regulators (43).

\section{CONCLUSIONS}

Cranberry juice has been long recognized for its protective effects in urinary tract infections. As evidenced by scores of published literature ingestion of cranberry juice may provide therapeutic benefits for other ailments. Given the risk involved in food-drug interaction, as exemplified by grapefruit juice, it is always prudent to understand the probable mechanism(s) 
Table 2. Effects on warfarin pharmacodynamics by cranberry juice

\begin{tabular}{|c|c|c|}
\hline Study type & Participants & Key Observations \\
\hline $\begin{array}{l}14 \text { days pre-treatment, t.i.d CJ / } \\
\text { single high dose of warfarin (26) }\end{array}$ & Healthy subjects $(\mathrm{n}=12)$ & Significant change in INR $(30 \%)$ \\
\hline $\begin{array}{l}5 \text { days pre-treatment, t.i.d CJ / single } \\
\text { dose of warfarin (23) }\end{array}$ & Healthy subjects $(\mathrm{n}=10)$ & $\begin{array}{l}\text { Thromboplastin time (area under } \\
\text { curve) and Emax of thromboplastin } \\
\text { time was not altered }\end{array}$ \\
\hline $\begin{array}{l}14 \text { day pre-treatment once daily CJ / } \\
\text { stable dose of warfarin }(24)\end{array}$ & Patients ( $\mathrm{n}=14 \mathrm{CJ} ; \mathrm{n}=16$ placebo) & $\begin{array}{l}\text { INR was not influenced by CJ } \\
\text { during the various points during / } \\
\text { after study. However, on day } 12 \text { a } \\
\text { small but statistically significant } \\
\text { change was noted }\end{array}$ \\
\hline $\begin{array}{l}7 \text { day pre-treatment, b.i.d CJ / stable } \\
\text { dose of warfarin }(25)\end{array}$ & Patients $(\mathrm{n}=10)$ & $\begin{array}{l}\text { INR and thrombin times were not } \\
\text { impacted during the various time } \\
\text { points in the trail }\end{array}$ \\
\hline $\begin{array}{l}7 \text { days pre-treatment once daily CJ / } \\
\text { stable dose of warfarin (27) }\end{array}$ & Patients $(\mathrm{n}=7)$ & $\begin{array}{l}\text { INRs remained stable as compared } \\
\text { to baseline values during the entire } \\
\text { trail }\end{array}$ \\
\hline
\end{tabular}

CJ: cranberry juice

for herb-drug interaction and examine the evidence to obtain guidance for the use of cranberry juice as a herbal supplement for dietary or nutritional needs.Cranberry juice has the potential to inhibit CYP enzymes (CYP3A and CYP2C9) under in vitro conditions, and at higher tested amounts of the juice the extent of inhibition was almost similar to the potent inhibitors of CYP3A (ketoconazole) and CYP2C9 (fluconazole). However, in relation to other beverages such as grape juice, tea and pomegranate juice, it appeared that cranberry juice was less potent in its CYP2C9 inhibitory properties, but the CYP3A inhibition was in line with or superior to grapefruit juice or pomegranate juice. In spite of the in vitro CYP inhibitions or Pgp inhibition produced by cranberry juice, there was no clinical translatability of the evidence in the pharmacokinetic disposition of scores of substrates (cyclosporine, warfarin, diclofenac, flurbiprofen, tizanidine, amoxicillin, ceflacor) with the exception of a single report on midazolam, where there was a moderate increase in the exposure of midazolam in subjects pretreated with cranberry juice. Interestingly, another study concluded lack of clinically relevant in vivo interaction between cranberry juice and midazolam. In general, the lack of in vivo translation of the in vitro CYP inhibition by cranberry juice was evident with the following speculative remarks: a) under in vitro conditions all anthocyanin principles may be available to have a concerted effort in CYP inhibition; however, limited anthocyanin principles at low levels were bioavailable in the in vivo studies; $b$ ) a faster clearance of the active anthocyanin principles under in vivo conditions may have led to levels not reaching the threshold level to show CYP inhibition; c) influence of protein binding and/or rapid tissue uptake of the substrate may be relevant under in vivo conditions, thereby limiting the drug availability to the enzymes.

It is noteworthy to provide some conclusive remarks with respect to pharmacodynamics of warfarin after ingestion of cranberry juice. While the debate continues on the issue of a pharmacodynamic interaction between warfarin and cranberry juice, the summation of the pharmacodynamics data obtained in patients and healthy subjects from the various prospective and controlled clinical trials do not provide overwhelming support for a pharmacodynamic drug interaction with cranberry juice. However, it was apparent that consumption of large quantity of cranberry juice (about 1-2 L per day) or concentrate $(1000 \mathrm{mg})$ for a longer duration of time (>3-4 weeks) may be temporally correlated with altered INR values. Therefore, while the total avoidance of cranberry juice by warfarin users may not be warranted by the published studies, in certain situations of higher intake of cranberry juice or concentrate there may be a need to monitor both warfarin doses and INR levels.

\section{ACKNOWLEDGEMENTS}

The author has no conflicts of interest to report. 


\section{REFERENCES}

1) Howell $\mathrm{AB}$. Cranberry proanthocyanidins and the maintenance of urinary tract health. Crit Rev Food Sci Nutr 2002; 42: 273-8.

2) Greenberg JA, Newmann SJ, Howell AB. Consumption of sweetened dried cranberries versus unsweetened raisins for inhibition of uropathogenic Escherichia coli adhesion in human urine: a pilot study. J Altern Complement Med 2005; 11: 875-8.

3) Nowack R. Cranberry juice - a well-characterized folk-remedy against bacterial urinary tract infection. Wien Med Wochenschr 2007; 157: 325 30 .

4) Rossi R, Porta S, Canovi B. Overview on cranberry and urinary tract infections in females. $\mathrm{J}$ Clin Gastroenterol 2010; 44: S61-2.

5) Ruel G, Lapointe A, Pomerleau S, Couture P, Lemieux S, Lamarche B, Couillard C. Evidence that cranberry juice may improve augmentation index in overweight men. Nutr Res 2013; 33: 419.

6) Shidfar F, Heydari I, Hajimiresmaiel SJ, Hosseini S, Shidfar S, Amiri F. The effects of cranberry juice on serum glucose, apoB, apoA-I, Lp(a), and Paraoxonase- 1 activity in type 2 diabetic male patients. J Res Med Sci 2012; 17: 355-60.

7) Shmuely H, Ofek I, Weiss EI, Rones Z, HouriHaddad Y. Cranberry components for the therapy of infectious disease. Curr Opin Biotechnol 2012; 23: $148-52$.

8) Bodet C, Grenier D, Chandad F, Ofek I, Steinberg D, Weiss EI. Potential oral health benefits of cranberry. Crit Rev Food Sci Nutr 2008; 48: 67280 .

9) Dohadwala MM, Holbrook M, Hamburg NM, Shenouda SM, Chung WB, Titas M, Kluge MA, Wang N, Palmisano J, Milbury PE, Blumberg JB, Vita JA. Effects of cranberry juice consumption on vascular function in patients with coronary artery disease. Am J Clin Nutr 2011; 93: 934-40.

10) Heuberger R. Polypharmacy and food-drug interactions among older persons: a review. J Nutr Gerontol Geriatr 2012; 31: 325-403.

11) Mani S, Ghalib M, Chaudhary I, Goel S. Alterations of chemotherapeutic pharmacokinetic profiles by drug-drug interactions. Expert Opin Drug Metab Toxicol 2009; 5 :109-30.

12) Dovjak P. Tools in polypharmacy. Current evidence from observational and controlled studies. Z Gerontol Geriatr. 2012; 45: 468-72.

13) Grace MH, Massey AR, Mbeunkui F, Yousef GG, Lila MA. Comparison of health-relevant flavonoids in commonly consumed cranberry products. J Food Sci 2012; 77:H176-83.

14) Dolton MJ, Roufogalis BD, McLachlan AJ. Fruit juices as perpetrators of drug interactions: the role of organic anion-transporting polypeptides. Clin Pharmacol Ther 2012; 92: 622-30.
15) Won CS, Oberlies NH, Paine MF. Influence of dietary substances on intestinal drug metabolism and transport. Curr Drug Metab 2010; 11: 778-92.

16) Hanley MJ, Cancalon P, Widmer WW, Greenblatt DJ. The effect of grapefruit juice on drug disposition. Expert Opin Drug Metab Toxicol 2011; 7:267-86.

17) Cuciureanu M, Vlase L, Muntean D, Varlan I, Cuciureanu R. Grapefruit juice--drug interactions: importance for pharmacotherapy. Rev Med Chir Soc Med Nat Iasi 2010; 11: 885-91

18) Ngo N, Yan Z, Graf TN, Carrizosa DR, Kashuba $\mathrm{AD}$, Dees EC, Oberlies NH, Paine MF. Identification of a cranberry juice product that inhibits enteric CYP3A-mediated first-pass metabolism in humans. Drug Metab Dispos 2009; 37: 514-22.

19) Ngo N, Brantley SJ, Carrizosa DR, Kashuba AD, Dees EC, Kroll DJ, Oberlies NH, Paine MF. The warfarin-cranberry juice interaction revisited: A systematic in vitro-in vivo evaluation. J Exp Pharmacol 2010; 2010: 83-91.

20) Ushijima K, Tsuruoka S, Tsuda H, Hasegawa G, Obi Y, Kaneda T, Takahashi M, Maekawa T, Sasaki T, Koshimizu TA, Fujimura A. Cranberry juice suppressed the diclofenac metabolism by human liver microsomes, but not in healthy human subjects. Br J Clin Pharmacol 2009; 68: 194-200.

21) Greenblatt DJ, von Moltke LL, Perloff ES, Luo Y, Harmatz JS, Zinny MA. Interaction of flurbiprofen with cranberry juice, grape juice, tea, and fluconazole: in vitro and clinical studies. Clin Pharmacol Ther 2006; $79: 125-33$.

22) Uesawa Y, Mohri K. Effects of cranberry juice on nifedipine pharmacokinetics in rats. $\mathrm{J}$ Pharm Pharmacol 2006; 58: 1067-72.

23) Lilja JJ, Backman JT, Neuvonen PJ. Effects of daily ingestion of cranberry juice on the pharmacokinetics of warfarin, tizanidine, and midazolam--probes of CYP2C9, CYP1A2, and CYP3A4. Clin Pharmacol Ther 2007; 81: 833-9.

24) Ansell J, McDonough M, Zhao Y, Harmatz JS, Greenblatt DJ. The absence of an interaction between warfarin and cranberry juice: a randomized, double-blind trial. J Clin Pharmacol 2009; 49: 824-30.

25) Mellen CK, Ford M, Rindone JP. Effect of highdose cranberry juice on the pharmacodynamics of warfarin in patients. Br J Clin Pharmacol 2010; 70: 139-42.

26) Mohammed Abdul MI, Jiang $X$, Williams KM, Day RO, Roufogalis BD, Liauw WS, Xu H, McLachlan AJ. Pharmacodynamic interaction of warfarin with cranberry but not with garlic in healthy subjects. Br J Pharmacol 2008; 154: 1691700 .

27) Li Z, Seeram NP, Carpenter CL, Thames G, Minutti C, Bowerman S. Cranberry does not affect prothrombin time in male subjects on warfarin. $\mathrm{J}$ Am Diet Assoc 2006; 106: 2057-61. 
28) Li M, Andrew MA, Wang J, et al. Effects of cranberry juice on pharmacokinetics of betalactam antibiotics following oral administration. Antimicrob Agents Chemother 2009; 53: 2725-32.

29) Grenier J, Fradette C, Morelli G, Merritt GJ, Vranderick M, Ducharme MP. Pomelo juice, but not cranberry juice, affects the pharmacokinetics of cyclosporine in humans. Clin Pharmacol Ther 2006; 79: 255-62.

30) Duthie GG, Kyle JA, Jenkinson AM, Duthie SJ, Baxter GJ, Paterson JR. Increased salicylate concentrations in urine of human volunteers after consumption of cranberry juice. J Agric Food Chem 2005; 53: 2897-900.

31) Milbury PE, Vita JA, Blumberg JB. Anthocyanins are bioavailable in humans following an acute dose of cranberry juice. J Nutr 2010; 140: 1099 . 104.

32) Kupferschmidt HH, Ha HR, Ziegler WH, Meier $\mathrm{PJ}$, Krahenbuhl S. Interaction between grapefruit juice and midazolam in humans. Clin Pharmacol Ther 1995; 58: 20-8.

33) Andersen V, Pedersen N, Larsen NE, Sonne J, Larsen S. Intestinal first pass metabolism of midazolam in liver cirrhosis --effect of grapefruit juice. Br J Clin Pharmacol 2002; 54: 120-4.

34) Yee GC, Stanley DL, Pessa LJ, Dalla Costa T, Beltz SE, Ruiz J, Lowenthal DT. Effect of grapefruit juice on blood cyclosporin concentration. Lancet. 1995; 345: 955-6.

35) Ducharme MP, Warbasse LH, Edwards DJ. Disposition of intravenous and oral cyclosporine after administration with grapefruit juice. Clin Pharmacol Ther 1995; 57: 485-91.

36) $\mathrm{Ku}$ YM, Min DI, Flanigan M. Effect of grapefruit juice on the pharmacokinetics of microemulsion cyclosporine and its metabolite in healthy volunteers: does the formulation difference matter? J Clin Pharmacol 1998; 38: 959-65.
37) Kim E, Sy-Cordero A, Graf TN, Brantley SJ, Paine MF, Oberlies NH. Isolation and identification of intestinal CYP3A inhibitors from cranberry (Vaccinium macrocarpon) using human intestinal microsomes. Planta Med 2011; 77: 26570 .

38) Suvarna R, Pirmohamed M, Henderson L. Possible interaction between warfarin and cranberry juice. BMJ 2003; 327: 1454.

39) Grant P. Warfarin and cranberry juice: an interaction? J Heart Valve Dis 2004; 13: 25-6.

40) Rindone JP, Murphy TW. Warfarin-cranberry juice interaction resulting in profound hypoprothrombinemia and bleeding. Am J Ther 2006; 13: 283-4.

41) Paeng $\mathrm{CH}$, Sprague $\mathrm{M}$, Jackevicius CA. Interaction between warfarin and cranberry juice. Clin Ther 2007; 29: 1730-5.

42) Welch JM, Forster K. Probable elevation in international normalized ratio from cranberry juice. J Pharm Technol 2007; 23: 104-7.

43) Zikria J, Goldman R, Ansell J. Cranberry juice and warfarin: when bad publicity trumps science. Am J Med 2010; 123: 384-92.

44) Hidaka M, Okumura M, Fujita K, Ogikubo T, Yamasaki K, Iwakiri T, Setoguchi N, Arimori K. Effects of pomegranate juice on human cytochrome p450 3A (CYP3A) and carbamazepine pharmacokinetics in rats. Drug Metab Dispos 2005; 33:644-8.

45) Nagata M, Hidaka M, Sekiya H, Kawano Y, Yamasaki K, Okumura M, Arimori K.Effects of pomegranate juice on human cytochrome P450 2C9 and tolbutamide pharmacokinetics in rats. Drug Metab Dispos. 2007; 35: 302-5. 